\title{
Estratégias de Educação Permanente na Avaliação das Equipes de Saúde da Família: uma Revisão Sistemática
}

\author{
Permanent Education Strategies in the Evaluation of Family \\ Health Teams: a Systematic Review
}

\author{
Livia Mendes Mesquita ${ }^{\mathrm{I}}$ (1D) \\ Geilsa Soraia Cavalcanti Valente ${ }^{1}$ (iD \\ Raquel Lima Soeiro ${ }^{\mathrm{I}}$ (D) \\ Elaine Antunes Cortez ${ }^{\mathrm{I}}$ (iD \\ Bianca Maria Innocencio da Silveira Lobo ${ }^{\mathrm{I}}$ (iD \\ Simone Costa da Matta Xavier ${ }^{1}$ iD
}

\section{PALAVRAS-CHAVE}

- Estratégia Saúde da Família.

- Atenção Básica.

- Educação Permanente.

- Avaliação em Saúde.

- Gestão da Qualidade em Saúde.

O Programa de Melhoria de Acesso e Qualidade da Atenção Básica, implantado pelo Ministério da Saúde, visa garantir a melhoria do acesso e da qualidade dos serviços ofertados pela atenção básica. Essa estratégia tem como perspectiva a promoção de melhorias do processo de trabalho e da assistência à saúde com base na indução, no monitoramento e na avaliação de processos e resultados a serem alcançados pelas equipes da Estratégia Saúde da Família. Nesse contexto, apresenta como uma das suas ferramentas mais potentes a educação permanente em saúde, que tem como objetivo promover as mudanças das práticas de atenção, gestão, educação e participação. Este artigo objetiva descrever as estratégias de educação permanente em saúde utilizadas no processo de avaliação das equipes de saúde da família, visando à melhoria da qualidade da assistência. Trata-se de uma revisão sistemática, com vistas a responder à seguinte questão de pesquisa: "Quais estratégias devem ser inseridas no âmbito das equipes de saúde da família para consolidar os processos avaliativos fomentados pelo Programa Nacional de Melhoria do Acesso e da Qualidade da Atenção Básica, tendo como foco a educação permanente em saúde?". Em síntese, os artigos selecionados nesta revisão apresentam as experiências em relação à avaliação do Programa Nacional de Melhoria do Acesso e da Qualidade da Atenção Básica, as ações referentes à educação permanente em saúde inseridas no processo de trabalho das equipes de saúde da família e o grau de incorporação de tecnologias de informação e comunicação na atenção básica. Ainda há evidências do uso de práticas educativas formativas e verticalizadas que não levam em consideração as necessidades do território nem as demandas dos profissionais no cotidiano do trabalho em saúde. A revisão demonstra a existência de uma lacuna do conhecimento que retrate estratégias de educação permanente para efetivação do processo avaliativo fomentado pelo Programa de Melhoria de Acesso e Qualidade da Atenção Básica. 


\section{KEY-WORDS}

- Family Health Strategy.

- Primary Health Care.

- Continuing Education.

- Health Evaluation.

- Quality Management.

\section{ABSTRACT}

The Program for Primary Care Access and Quality Improvement, implemented by the Ministry of Health, aims to ensure the improvement of access and quality of services offered by Primary Care. This strategy aims to promote work process and health care improvements based on the induction, monitoring and evaluation of processes and results to be achieved by the Family Health Strategy teams. In this context, one of its most potent tools is permanent health education, which aims to promote changes in care practices, management, education and participation. This article aims to describe the Permanent Health Education strategies used in the evaluation process of family health teams, aiming at improving the quality of care. This is a systematic review, aimed at answering the following research question: "What strategies should be included within the scope of family health teams to consolidate the evaluation processes promoted by the National Program for Primary Care Access and Quality Improvement, focusing on Permanent Health Education?". In summary, the articles selected for this review depict the experiences regarding the evaluation of the National Program for Primary Care Access and Quality Improvement, the actions related to Permanent Health Education included in the work process of family health teams and the degree of incorporation of information and communication technologies into primary care. There is still evidence on the use of formative and verticalized educational practices that do not take into account the needs of the territory or the demands of professionals in the health care daily work. The review demonstrates the existence of a knowledge gap that portrays continuing education strategies for the effectiveness of the evaluation process promoted by the Program for Primary Care Access and Quality Improvement.

Recebido em 2/9/19

Aceito em 8/10/19

\section{INTRODUÇÃO}

Com a expansão das políticas públicas de atenção à saúde e a consolidação da Estratégia Saúde da Família (ESF) como modelo prioritário para reorganização da atenção primária à saúde (APS), a questão da qualidade da gestão e das práticas das equipes de atenção básica $(\mathrm{AB})$ assume um importante papel no cenário nacional. Diante disso, o governo federal passa a centrar suas iniciativas na qualificação das ações e na efetividade das intervenções em saúde da AB. Sendo assim, reconhecendo a importância de qualificar o acesso e o cuidado prestado na AB, o Ministério da Saúde (MS) lançou, em 2011, o Programa Nacional de Melhoria do Acesso e da Qualidade da Atenção Básica, o PMAQ, que simboliza um importante avanço no processo de negociação e pactuação entre os três níveis de governo ${ }^{1-3}$.

Instituído pela Portaria no ${ }^{\circ} .654 / 2011$ do MS, o PMAQ visa ampliar a oferta qualificada dos serviços de saúde no âmbito do Sistema Único de Saúde (SUS) e institucionalizar a cultura da avaliação na atenção básica em saúde $(\mathrm{ABS})^{3}$. O programa dispõe de um conjunto de estratégias de qualificação, acompanhamento e avaliação do trabalho das equipes de saúde. De acordo com o novo desenho proposto pela Portaria 1.645, de 2 de outubro de 2015, está estruturado em três fases distintas (adesão e contratualização, certificação e recontratualização) e um eixo estratégico transversal de desenvolvimento. Os processos de autoavaliação, monitoramento, educação permanente (EP), apoio institucional e cooperação horizontal compõem o eixo estratégico transversal de desenvolvimento do PMAQ $^{4,5}$.

Esse eixo ordenador de ações é considerado como transversal, pois perpassa por todas as fases do programa. Nesse contexto, destaca-se como eixo estratégico do PMAQ, a EP que propõe transformar as práticas do saber e do fazer no trabalho, considerando o conhecimento prévio e as experiências dos indivíduos ${ }^{6}$. A EP, além da sua evidente dimensão pedagógica, deve ser encarada também como uma importante estratégia de gestão ${ }^{4}$, com grande potencial provocador de mudanças no cotidiano dos serviços, em sua micropolítica, próximo dos efeitos concretos das práticas de saúde na vida dos usuários, e como um processo que se dá "no trabalho, pelo trabalho e para o trabalho". Essa reflexão reforça a premissa de que a EP firma-se na construção de relações e processos que partem do interior das equipes de saúde em consonância às práticas organizacionais ${ }^{7}$. Ao repensarem e refletirem sobre suas práticas no cotidiano laboral, é possível que as equipes de saúde identifiquem as principais necessidades da população atendida e os nós críticos em relação ao acesso ${ }^{7,8}$.

Partindo desse pressuposto, as práticas avaliativas devem ser incorporadas ao processo de trabalho em saúde utilizando o eixo estratégico da EP como dispositivo para planejamento e programação das ações coletivas e no conhecimento dos problemas e das necessidades de saúde apresentados pela população ${ }^{9}$. A avaliação não deve ser encarada como algo punitivo, e sim como um importante fomentador de mudanças para a tomada de decisões e para melhoria do desempenho da equipe, resultando assim em serviços de qualidade ${ }^{6,10,11}$. Diante desse contexto, a partir das experiências vivenciadas nesse processo de avaliação do MS, o presente estudo teve como objetivo descrever as estratégias inseridas no âmbito das equipes de saúde da família (EqSF) para consolidar os processos avaliativos fomentados pelo PMAQ tendo como foco a educação permanente em saúde (EPS).

\section{MÉTODO}

Trata-se de uma revisão sistemática da literatura que utiliza métodos sistemáticos para identificar, selecionar, avaliar criticamente e sintetizar os estudos mais relevantes e suas respectivas contribuições a partir de uma pergunta formulada de forma clara e bem definida. A metodologia para o desenvolvimento de uma revisão sistemática de literatura está pautada na: 1. elaboração de uma questão de pesquisa orientadora da estratégia de busca; 2 . busca na literatura - variedade de fontes para a localização dos estudos; 3. seleção dos artigos - definição de critérios de inclusão e 
exclusão; 4. extração dos dados; 5. avaliação da qualidade metodológica das produções recuperada; 6. síntese dos dados (metanálise); 7. avaliação da qualidade das evidências; e 8. redação e publicação dos resultados ${ }^{12,13}$.

A revisão sistemática é classificada como uma modalidade de revisão que caracteriza-se por trazer informações gerais sobre o tema abordado, sendo considerada o melhor nível de evidência para tomadas de decisão. Geralmente, por seguir um rigor metodológico e apresentar um resultado novo, é conceituada como uma contribuição original, permitindo assim que outros pesquisadores possam repetir o procedimento ${ }^{12}$.

A revisão foi realizada por meio do levantamento de material científico no período de agosto a dezembro de 2018, com vistas a responder à seguinte questão de pesquisa:

- Quais estratégias devem ser inseridas no âmbito das EqSF para consolidar os processos avaliativos fomentados pelo PMAQ, tendo como foco a EPS?

A partir da pergunta de pesquisa, foram definidos os descritores a serem utilizados e suas combinações nas línguas portuguesa e inglesa, utilizando a estratégia PICO, sendo estes: P (população ou problema) - Estratégia Saúde da Família (ESF) ou atenção básica (AB) - atenção primária à saúde (APS); I (intervenção proposta) - educação permanente (EP) - educação continuada (EC); C constitui controle ou comparação, porém não cabe para este estudo; O (desfecho, resultado esperado) - avaliação em saúde e gestão da qualidade em saúde. E como MeSH terms: Family Health Strategy or Primary Health Care, Continuing Education, Health Evaluation and Quality Management.

A busca on-line por artigos originais foi realizada na Scopus via Portal Capes e no portal da Biblioteca Virtual em Saúde (BVS), nas bases de dados: Literatura Latino-Americana e do Caribe em Ciências da Saúde (Lilacs), Base de Dados em Enfermagem (BDENF), Índice Bibliográfico Español em Ciencias de la Salud (Ibecs), Medline no Portal US National Library of Medicine e PubMed com o objetivo de apresentar um panorama da produção científica sobre o tema.

Para o estabelecimento dos critérios de inclusão dos estudos, foram levados em consideração artigos originais e publicados na íntegra que estivessem disponíveis nas bases de dados nos últimos cinco anos, nos idiomas português, espanhol e inglês, relacionados à temática. Os critérios de exclusão foram os seguintes: trabalhos de conclusão de curso de graduação, monografias de especialização, dissertações e/ou teses e relatórios de pesquisa. Para a identificação dos estudos, realizou-se a leitura criteriosa dos títulos, dos resumos e das palavras-chave de todas as publicações completas localizadas pela estratégia de busca. Os artigos que após a leitura dos resumos não contemplassem a temática proposta foram excluídos, assim como os que se encontravam em duplicidade.

A associação dos descritores, utilizando o booliano AND e OR, teve como objetivo buscar o refinamento dos estudos. Respeitando os critérios de inclusão e exclusão, atingiu-se o total de 36 trabalhos. De acordo com proposta do projeto e a abordagem da pesquisa, foram selecionados sete artigos considerados relevantes para o estudo. Utilizaram-se as seguintes associações: Estratégia Saúde da Família OR Atenção Básica $A N D$ Educação Permanente AND Avaliação em saúde; Estratégia Saúde da Família OR Atenção Básica AND Educação Permanente AND Gestão da Qualidade em saúde; Avaliação em saúde $O R$ Gestão da qualidade em saúde $A N D$ Educação Permanente; Avaliação em saúde $O R$ Gestão da qualidade em saúde AND Estratégia Saúde da Família OR Atenção Básica;
Avaliação em saúde $O R$ Gestão da qualidade em saúde $A N D$ Educação Permanente AND Estratégia Saúde da Família $O R$ Atenção Básica. As estratégias de busca em inglês foram utilizadas de maneira similar.

No cruzamento dos termos "Avaliação em Saúde $O R$ Gestão da Qualidade em saúde AND Educação Permanente", foram encontrados os mesmos estudos da associação realizada com os descritores "Avaliação em Saúde OR Gestão da qualidade em saúde AND Educação Permanente AND Estratégia Saúde da Família OR Atenção Básica”. Na Figura 1, podem-se observar o caminho metodológico percorrido para a escolha dos artigos por meio dos descritores associados e o quantitativo de produções bibliográficas a serem analisadas.

Após a seleção dos artigos de acordo com a temática previamente definida, os estudos foram analisados detalhadamente de forma crítica. Selecionaram-se sete artigos para compor a análise e a categorização propostas neste trabalho. As informações extraídas de cada artigo foram categorizadas em um quadro sinóptico, em ordem cronológica, apresentando as características e as principais conclusões de cada estudo.

\section{RESULTADOS}

A análise dos artigos fez emergir as seguintes categorias: "PMAQ: análise das experiências no processo de avaliação", "Estratégias de educação permanente em saúde: a busca pela melhoria da qualidade da assistência" e "Incorporação das tecnologias de informação e comunicação (TIC) na atenção básica”.

\section{PMAQ: análise das experiências no processo de avaliação}

Dois dos artigos selecionados ${ }^{14,18}$ retratam a experiência no processo de implementação do PMAQ. O artigo "PMAQ-AB: a experiência local para a qualificação do Programa Nacional” discorre sobre a implantação do PMAQ no estado da Paraíba a partir da vivência da equipe de avaliação externa, apontando aspectos importantes do desenvolvimento do programa nos três ciclos realizados. Segundo os autores, todos os profissionais envolvidos são protagonistas na estruturação do processo, no entanto verificou-se ainda que algumas equipes da $\mathrm{AB}$ não se sentiram envolvidas em todas as fases do programa. Apesar de a adesão ao programa ter sido considerada bastante positiva, ainda parece evidenciar modos de organização dos serviços, nos quais os trabalhadores são entendidos como meros implementadores de ações. O estudo ressalta que para alguns profissionais a avaliação externa do PMAQ ainda é vista com caráter de punição, gerando posturas negativas ao processo.

$\mathrm{O}$ outro artigo ${ }^{14}$ que trata da implementação do PMAQ traz a lógica desse processo segundo os gestores da $\mathrm{AB}$ de São Paulo. Por meio da avaliação desses profissionais, foi possível perceber que muitos ainda apresentam uma concepção sobre o processo de cunho fiscalizatório, o que corrobora o artigo citado anteriormente. Entretanto, alguns atores versaram sobre as contribuições do programa na organização dos processos de trabalho das EqSF e na própria atuação como gestor na $\mathrm{AB}$. Segundo os gerentes, houve uma mudança significativa referente ao processo considerado no primeiro ciclo como gerador de maior carga de trabalho. Os entrevistados revelaram que o processo de avaliação pode ser visto como um momento de reflexão crítica sobre o trabalho realizado, apesar de ainda incipiente, direcionando a prática e colaborando no planejamento estratégico das equipes. Os autores reiteram a necessidade de aprimoramento do trabalho por meio da discussão de indicadores e do incentivo à qualificação dos profissionais por meio da EP e de melhorias estruturais.

REVISTA BRASILEIRA DE EDUCAÇÃO MÉDICA

3 44(1) : e010; 2020 


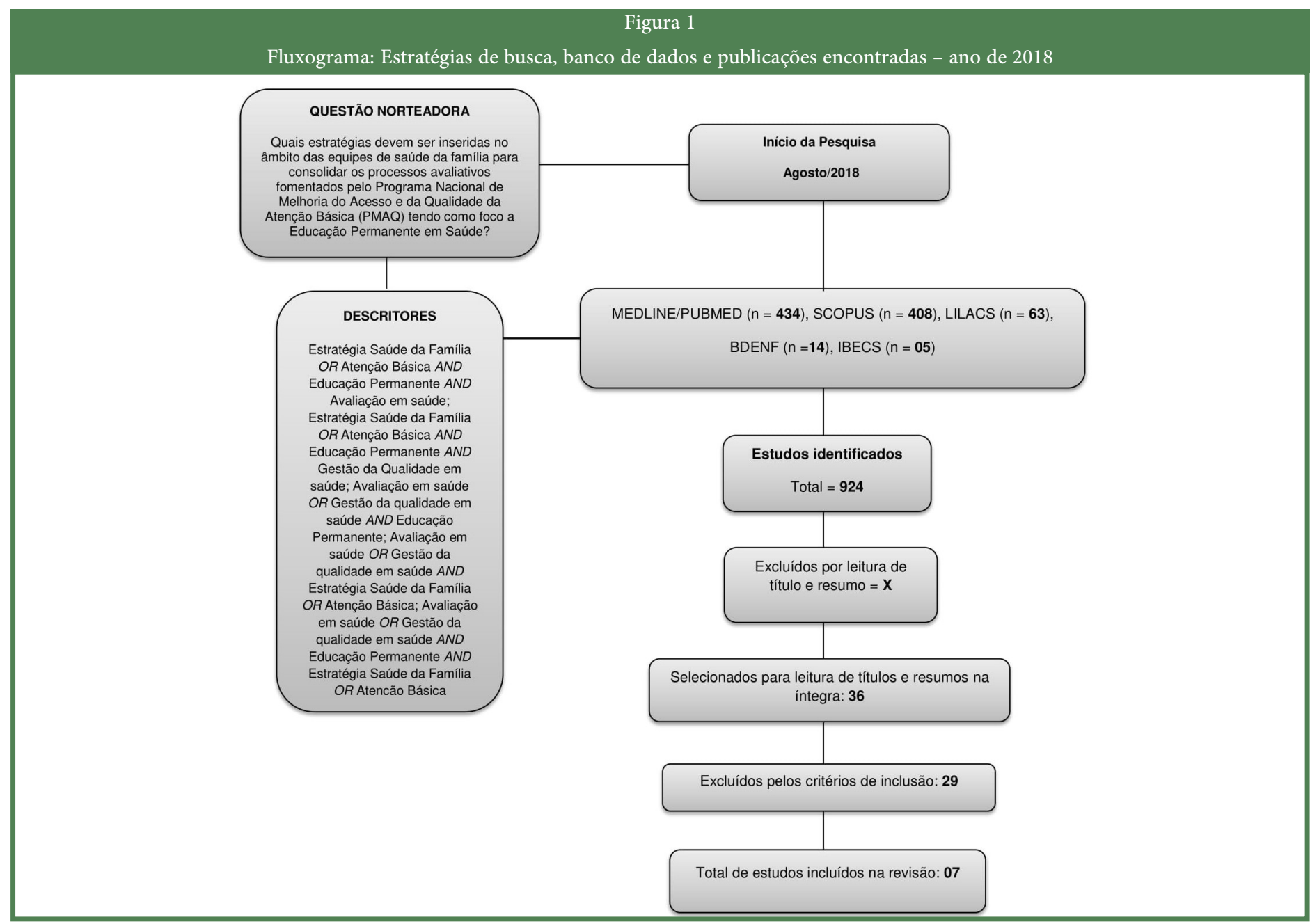

Fonte: Elaborado pelas autoras.

Estratégias de educação permanente em saúde: a busca pela melhoria da qualidade da assistência

No artigo "Educação permanente em saúde: uma estratégia para refletir sobre o processo de trabalho", os autores ${ }^{17}$ relatam todo o planejamento para execução de uma atividade de EP desenvolvida para odontólogos da ESF no município de Fortaleza/CE, pautada na metodologia problematizadora segundo os princípios teóricos de Paulo Freire. Dentro das questões discutidas, $\mathrm{o}$ acesso e a qualidade aos serviços de saúde trazidos pelo PMAQ foram colocados em pauta. A intenção foi despertar os profissionais a refletir sobre seus processos de trabalho desenhando formas de fazer baseadas nas necessidades dos usuários. Foi ressaltada a importância do PMAQ para o aperfeiçoamento das ações desenvolvidas pelos serviços, destacando que a continuidade do processo suscitado pelo programa é imprescindível para a melhoria da qualidade da APS.

O estudo ${ }^{19}$ realizado em Mato Grosso do Sul apresenta uma análise na perspectiva das equipes que participaram do primeiro ciclo do programa em consonância com as ações de EP. De acordo com os autores, a reponsabilidade com a qualidade da assistência à população conduziu a adesão do estado ao PMAQ, pressupondo estratégias integradas entre as esferas municipais e estaduais. $\mathrm{O}$ apoio da gestão foi considerado um diferencial para o planejamento e a organização do processo de trabalho, dinamizando as ações de EPS. Os resultados do estudo evidenciaram a realização de cursos presenciais pelas EqSF como as ações mais implementadas no que se refere à $\mathrm{EP}$, demonstrando a necessidade de um aprofundamento maior da proposta da EPS a fim de repensar práticas educativas ainda tradicionais. As demais ações mencionadas no estudo como troca de experiências, teleducação, ensino a distância $(\mathrm{EaD})$ e tutoria/preceptoria também merecem destaque; em particular, ressaltam-se as TIC virtuais como estratégias potentes de ampliação do acesso à educação, porém ainda pouco utilizadas nas regiões mais distantes do estado.

$\mathrm{O}$ artigo anterior faz uma análise das características e da prevalência das ações de EP utilizadas pelas EqSF que aderiram ao PMAQ no primeiro ciclo. Já o estudo realizado nos municípios do estado da Bahia ${ }^{16}$ faz considerações referentes ao processo de EP na lógica do apoio matricial e como essa ferramenta está inserida nas práticas dos Núcleos de Apoio à Saúde da Família (Nasf) e das EqSF. Por meio do apoio matricial instituído com a criação dos Nasf, é possível a construção de espaços de reflexão e de compartilhamento de saberes, visando à qualificação do processo de trabalho das equipes.

Verificou-se, por meio dos resultados do estudo, que ainda há uma compreensão deturpada em relação à EPS. Cursos, treinamentos e capacitações pautados num modelo verticalizado de transmissão de informação são os reconhecidos pelos profissionais como práticas educativas, sendo muitas vezes realizados fora do espaço de trabalho. 


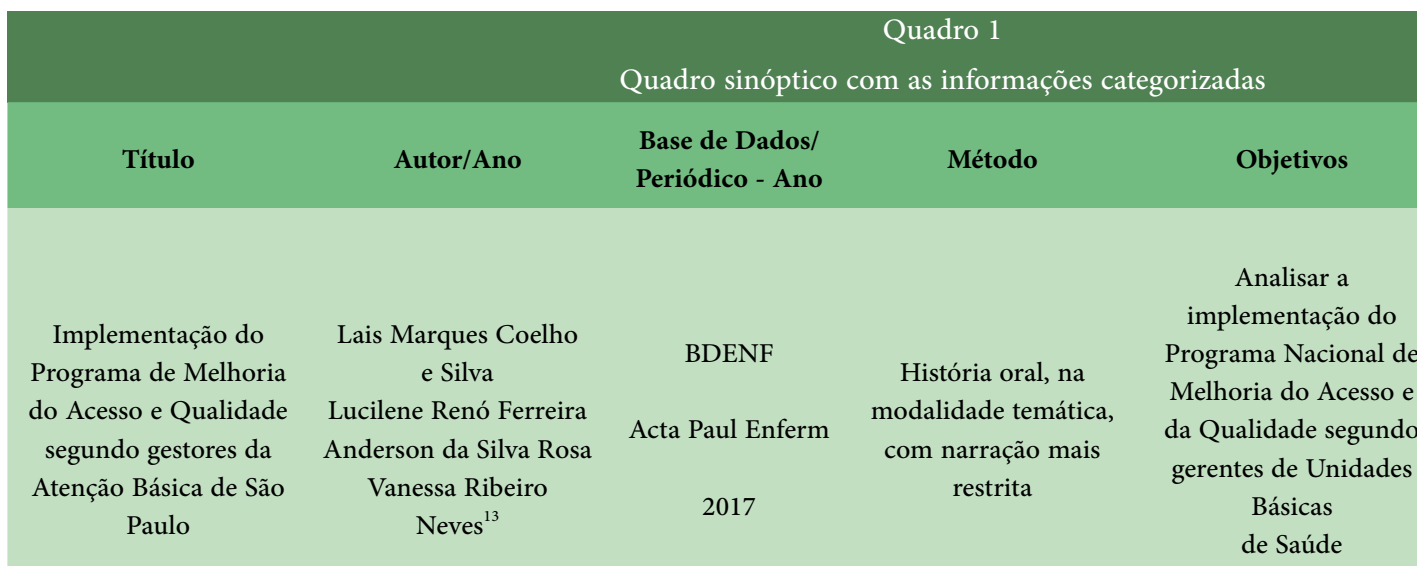

Alaneir F. dos Santos

Délcio F. Sobrinho;

Lucas L. Araujo;

Cristiane da S. Diniz Procópio;

Incorporação

de Tecnologias

de Informação e

Comunicação e

qualidade na atenção

básica em saúde no Brasil

Érica A. S. Lopes;

\section{MEDLINE}

Cad. Saúde Pública Estudo transversal

2017
Descrever a incorporação de TIC na atenção básica

e sua associação com

a qualidade, utilizando

Programa Nacional de

Melhoria do Acesso e da

Qualidade da Atenção Básica (PMAQ).
Angela Maria de

Lourdes D. de Lima

Clarice M. R. dos Reis;

Daisy Maria X. de

Abreu; Alzira Oliveira

Jorge;

Antonio Thomaz

Matta-Machado ${ }^{14}$
Educação permanente

e apoio matricial:

formação, vivências

e práticas dos

profissionais dos

Núcleos de Apoio à

Saúde da Família e das

equipes apoiadas

\section{José Patrício Bispo Júnior}

Diane Costa Moreira ${ }^{15}$

\section{PUBMED}

Cad. Saúde Pública

2017
Estudo de casos

múltiplos, com abordagem qualitativa

Compreender e analisar como os processos de educação permanente são vivenciados pelos profissionais dos Núcleos de Apoio à Saúde da Família (NASF) e das equipes de saúde da família (EqSF).

Compreender como o apoio matricial, como estratégia de educação permanente, estava incorporado ao trabalho desses profissionais.

Janaína Rocha de Sousa

Educação Permanente em Saúde: uma estratégia para refletir sobre o processo de trabalho

\section{Bizerril; Kátia de Góis}

Almeida; Davi Oliveira

Holanda Saldanha;

Maria Eneide Leitão de Almeida $^{16}$

Juliana Sampaio,

Marina Nascimento

PMAQ-AB: a experiência local para a qualificação do Programa Nacional de Moraes, Emanuella

de Castro Marcolino,

Israel Dias de Castro,

Luciano Bezerra

Gomes, Francisco de Sales Clementino ${ }^{17}$

\section{LILACS}

Metodologia problematizadora,

Revista da ABENO pautada no referencial teórico de Paulo

2016

Freire

Estudo descritivo

e analítico, tipo

documental dos

diários de campo

das equipes que

participaram da

Avaliação Externa do

PMAQ na PB
Induzir mudanças nas

diversas maneiras de

agir dos profissionais da

Estratégia de Saúde da

Família (ESF).

Conclusões

Evidenciou-se um processo incipiente de incorporação da cultura avaliativa de forma sistematizada e como subsídio para a melhoria contínua da qualidade na Atenção Básica. O PMAQ favoreceu a organização dos processos de trabalho e contribuiu para direcionar o olhar dos gestores à prática das equipes e à própria atuação.

O processo de incorporação de TIC está em curso no país, com uma parcela muito pequena das equipes de atenção básica possuindo um alto nível de incorporação.

Este estudo constatou ainda que existe associação entre a incorporação de tecnologias de informação e a qualidade da atenção observada nos resultados obtidos na certificação de qualidade do PMAQ.

Evidencia-se a não institucionalidade da educação permanente como política nos municípios estudados. A formação

sobre o apoio matricial e o processo de trabalho do NASF mostrou-se frágil para os grupos, o que interfere na função de apoio

e na gestão do cuidado.

Foi evidenciada a diminuta atuação dos NASF como promotores de educação permanente para as equipes apoiadas.

Obteve-se a construção do Manual de Promoção em Saúde Bucal e do Manual de Encaminhamentos para a Atenção Secundária em Odontologia

Avaliar o processo de implantação do PMAQ no Estado da Paraíba a partir da experiência da equipe de avaliação externa.
Ainda há um longo caminho para que as diretrizes do PMAQ sejam efetivadas na prática e que se torne um incentivo para a garantia do acesso e qualidade da atenção à saúde para a população brasileira. 


\begin{tabular}{|c|c|c|c|c|c|}
\hline & & & $\begin{array}{c}\text { Quadro } 1 \\
\text { Continuação. }\end{array}$ & & \\
\hline Título & Autor/Ano & $\begin{array}{l}\text { Base de Dados/ } \\
\text { Periódico - Ano }\end{array}$ & Método & Objetivos & Conclusões \\
\hline $\begin{array}{c}\text { Educação Permanente } \\
\text { no cotidiano da } \\
\text { Atenção Básica no } \\
\text { Mato Grosso do Sul }\end{array}$ & $\begin{array}{l}\text { Jacinta de Fátima } \\
\text { Franco Pereira } \\
\text { Machado, Alessandro } \\
\text { Diogo De Carli, } \\
\text { Vera Lúcia } \\
\text { Kodjaoglanian, Mara } \\
\text { Lisiane de Moraes dos } \\
\text { Santos }{ }^{19}\end{array}$ & SAÚDE DEBATE & $\begin{array}{l}\text { Estudo observacional } \\
\text { analítico transversal, } \\
\text { de ordem } \\
\text { multicêntrica, com } \\
\text { a participação de } \\
\text { Instituições de } \\
\text { Ensino e Pesquisa do } \\
\text { País e seguido pelo } \\
\text { Ministério da Saúde }\end{array}$ & $\begin{array}{l}\text { Analisar as ações de } \\
\text { Educação Permanente } \\
\text { em Saúde na Atenção } \\
\text { Básica, na perspectiva } \\
\text { de } 184 \text { equipes } \\
\text { participantes da primeira } \\
\text { fase do Programa de } \\
\text { Melhoria do Acesso e da } \\
\text { Qualidade (PMAQ) }\end{array}$ & $\begin{array}{l}\text { Os cursos presenciais foram mais } \\
\text { prevalentes, seguidos por troca de } \\
\text { experiência, teleducação, ensino } \\
\text { à distância e tutoria/preceptoria, } \\
\text { tanto na capital quanto no interior. } \\
\text { As ações de planejamento e apoio } \\
\text { à gestão foram mais prevalentes na } \\
\text { capital. As ações de apoio da gestão } \\
\text { estavam diretamente relacionadas } \\
\text { com o planejamento e a organização } \\
\text { do processo de trabalho. }\end{array}$ \\
\hline $\begin{array}{c}\text { Atenção Básica e } \\
\text { Educação Permanente } \\
\text { em Saúde: cenário } \\
\text { apontado pelo } \\
\text { Programa Nacional } \\
\text { de Melhoria do } \\
\text { Acesso e da Qualidade } \\
\text { da Atenção Básica } \\
\text { (PMAQ-AB) }\end{array}$ & $\begin{array}{c}\text { Hêider Aurélio Pinto, } \\
\text { Alcindo Antônio Ferla, } \\
\text { Ricardo Burg Ceccim, } \\
\text { Alexandre R. Florêncio, } \\
\text { Izabella Barison Matos, } \\
\text { Mirceli } \\
\text { Goulart Barbosa, Nilva } \\
\text { Lucia Rech Stédile, } \\
\text { Angelo Pagot Zortea }\end{array}$ & $\begin{array}{c}\text { LILACS } \\
\text { Revista Divulgação } \\
\text { em Saúde para } \\
\text { debate }\end{array}$ & $\begin{array}{l}\text { Análise descritiva, } \\
\text { com os dados } \\
\text { tratados segundo sua } \\
\text { frequência simples } \\
\text { e percentuais de } \\
\text { distribuição nos } \\
\text { diferentes quesitos de } \\
\text { resposta. }\end{array}$ & $\begin{array}{l}\text { Revisar as questões } \\
\text { relacionadas com a política } \\
\text { nacional de educação e } \\
\text { desenvolvimento para o } \\
\text { Sistema Único de Saúde } \\
\text { (SUS) e analisar questões } \\
\text { relativas ao ensino- } \\
\text { aprendizagem no cotidiano } \\
\text { da Atenção Básica, }\end{array}$ & $\begin{array}{l}\text { Concluiu-se que a EPS teve } \\
\text { expansão e abrangência importantes } \\
\text { na Atenção Básica; que as equipes } \\
\text { têm se conectado com ofertas } \\
\text { formativas; ainda há evidência } \\
\text { de processos educativos pontuais } \\
\text { e de cunho informacional; e que } \\
\text { as variações regionais de acesso } \\
\text { às tecnologias de informação e } \\
\text { comunicação são produzidas pelos } \\
\text { problemas de infraestrutura, não } \\
\text { pelo interesse e adesão. }\end{array}$ \\
\hline
\end{tabular}

Fonte: Elaborado pelas autoras.

Além disso, o estudo permitiu identificar que os conteúdos trabalhados versam sobre atualização de procedimentos, protocolos e rotinas baseados na metodologia da educação continuada, reforçando a necessidade de um maior aprofundamento sobre as diferenças dessas práticas de educação. Outro dado importante evidenciado pelos autores trata da formação referente ao apoio matricial. Os relatos dos profissionais dos Nasf e das EqSF revelaram a carência de informações sobre o sistema de apoio matricial e a restrita atuação do próprio núcleo como agente promotor de $\mathrm{EP}$, caracterizando uma fragilidade no que diz respeito à atuação desses sujeitos. O artigo ressalta a importância de um melhor entendimento sobre as ações de matriciamento e sua lógica de apoio técnico-pedagógica, além da premente necessidade de efetivação da política de EP a fim de transformar as práticas de trabalho das equipes que compõem a ESF.

Incorporação das tecnologias de informação e comunicação (TIC) na atenção básica

Outros autores ${ }^{15,20}$ descrevem em seus artigos estratégias educacionais pautadas na concepção da EP para fins de qualificação profissional e assistencial no âmbito da $\mathrm{AB}$.

$\mathrm{O}$ artigo que propõe a incorporação das $\mathrm{TIC}^{15}$ como forma de ampliar a qualidade do cuidado em saúde, utilizando como base o processo de avaliação incitado pelo PMAQ, analisou o grau de incorporação de tecnologias pelas equipes de $\mathrm{AB}$ no que se refere à infraestrutura, à implantação de sistemas e à utilização de informação. Os dados obtidos mostram que o processo de incorporação de tecnologias na área de saúde ainda possui limitações, como questões relacionadas à infraestrutura, aos entraves para implementação de políticas inovadoras nos ambientes institucionais e à influência de fatores individuais e profissionais. No entanto, observou-se que a utilização de informações no que tange aos indicadores para fins de planejamento e monitoramento de ações foi positiva, de acordo com o resultado da certificação das equipes de $\mathrm{AB}$ que aderiram ao PMAQ no primeiro ciclo, em todos os estados brasileiros. $\mathrm{Na}$ contramão, a avaliação correspondente à implantação de sistemas, ou seja, centrais de leito, regulação e exames, não avançou em suas dimensões assistenciais. Tendo em vista os aspectos examinados no estudo, há muito que se avançar em relação à associação das TIC ao cotidiano das equipes de $\mathrm{AB}$ no Brasil. Acredita-se que, uma vez incorporadas aos processos de planejamento de ações e considerando o registro adequado das informações, tende-se a obter melhorias na qualidade da atenção.

Em conformidade com o estudo apresentado, o artigo "Atenção básica e educação permanente em saúde: cenário apontado pelo Programa Nacional de Melhoria do Acesso e da Qualidade da Atenção Básica (PMAQ-AB) ${ }^{\prime 20}$ faz uma análise a partir dos dados obtidos no primeiro ciclo referente aos recursos de EPS utilizados pelas EqSF em todo território nacional. Ratificando as informações do estudo em Mato Grosso do Sul, constatou-se que o Telessaúde, os cursos presenciais e as trocas de experiências são as estratégias de ensino-aprendizagem no trabalho que apresentam maior aplicabilidade. Percebe-se que o acesso às TIC ainda era frágil em algumas regiões do país, demonstrando a necessidade de investimentos nessa área.

Os autores ${ }^{20}$ enfatizaram que, no processo de avaliação, não se contemplaram todas as formas de se fazer a EP, sendo fundamental 
ampliar o escopo da avaliação no que se refere às dinâmicas inseridas nas práticas cotidianas das equipes. $\mathrm{O}$ estudo revela também o papel fundamental do apoiador institucional para a qualificação do processo de trabalho aliada à EPS.

\section{DISCUSSÃO}

Em sua maioria, os estudos selecionado ${ }^{14-20}$ atendiam à temática do PMAQ e à questão da EP no contexto do processo de trabalho da $\mathrm{AB}$. No entanto, foram encontrados poucos artigos que trazem a discussão estratégias de EP que possam fomentar mudanças no cotidiano das EqSF a fim de estabelecer processos de avaliação para melhoria da qualidade da assistência, o que justifica o aprofundamento dessa questão.

$\mathrm{O}$ PMAQ-AB tem como objetivo principal desenvolver ações que assegurem maior acesso e qualidade aos serviços de saúde no SUS e tem como base estratégias de monitoramento e avaliação direcionadas à $\mathrm{AB}$. $\mathrm{O}$ ato de avaliar é, por vezes, caracterizado como algo que gera penalidade para aqueles que não alcançaram os resultados esperados, no entanto o programa traz como proposta os processos avaliativos, visando instrumentalizar as equipes da ESF para a tomada de decisões a partir da análise do processo de trabalho e da identificação das reais necessidades da população assistida por elas ${ }^{4}$.

$\mathrm{O}$ PMAQ-AB prioriza processos mais participativos, considerando todos os atores envolvidos com a $\mathrm{AB}$, ou seja, usuários, profissionais $\mathrm{e}$ gestores, reconhecendo-os como corresponsáveis pela qualificação do SUS. Para o processo em discussão, torna-se fundamental que todos os indivíduos implicados no fazer em saúde atuem de forma propositiva na construção de intervenções para enfrentamento dos problemas identificados, com vistas ao aprimoramento dos serviços ofertados ${ }^{21}$.

A EP configura-se como uma ferramenta de potencial pedagógico que possibilita a reorganização do processo de trabalho e a transformação do processo do cuidado pelos profissionais da saúde, uma vez que lhes permite refletir sobre suas práticas e empregar as mudanças necessárias que possam dinamizar o cotidiano dos serviços de saúde ${ }^{4,22}$.

A oferta de EPS aos profissionais contribui para maior qualificação do processo de trabalho, na medida em que estimula o desenvolvimento das competências do sujeito, produzindo mudanças de atitudes e ampliando as possibilidades para a superação das dificuldades vivenciadas pelos trabalhadores em seu cotidiano. Nesse sentido, o PMAQ preconiza que a gestão estimule processos de EP nas equipes para que se identifiquem as fragilidades e necessidades advindas do contexto local ${ }^{4,23}$.

Nessa perspectiva, considera-se a criação de espaços para a EP em saúde que sejam pautados em processos de ensino e aprendizagem crítico-reflexivos e participativos para que as ações e os serviços de saúde sejam mais efetivos.

Como eixo estratégico do PMAQ, a EP tem como pressuposto o aperfeiçoamento das ações desenvolvidas pelos serviços, estando diretamente relacionado ao processo de formação e desenvolvimento dos trabalhadores do SUS. Nesse aspecto, o apoio matricial se constitui um dispositivo ativo no processo de qualificação da assistência aos usuários e de capacidade de cuidado das equipes. Os Nasf-AB desempenham um trabalho técnico-pedagógico, produzindo ação de apoio educativo com e para as equipes, sendo a EPS uma de suas ferramentas no apoio às EqSF, visando ampliar e aperfeiçoar as práticas de gestão e de atenção à saúde, a partir das demandas cotidianas dos sujeitos que atuam na $\mathrm{AB}^{4,24}$.

$\mathrm{Na}$ avaliação do Nasf durante o processo do PMAQ, verifica-se o desenvolvimento de estratégias de EP na perspectiva da troca de saberes entre os diversos profissionais e da elaboração de projetos comuns de intervenção nas situações e nos problemas identificados num dado território ${ }^{24}$.

Nesse sentido, a proposta de EPS assume o compromisso de transformar as práticas do cotidiano do trabalho ao valorizar saberes e experiências dos profissionais, fortalecendo as formas de cuidado e produzindo avanços no que se refere à integralidade. A integralidade atua como norteadora da EPS, uma vez que envolve a compreensão dos problemas de saúde em suas diferentes dimensões e direciona o trabalho em saúde para um trabalho multidisciplinar e multiprofissiona ${ }^{23}$.

O PMAQ recomenda a incorporação das TIC como estratégias educativas vinculadas à EPS. A aproximação da educação com o trabalho por meio de dispositivos interativos possibilita o compartilhamento de diferentes saberes, o que leva ao aprimoramento dos profissionais de saúde. O conhecimento adquirido impacta a produção do cuidado, ampliando assim a resolubilidade clínica dos profissionais da $\mathrm{AB}$ e a capacidade de agir diante dos problemas identificados.

A estruturação do conhecimento deve ser orientada a partir da realidade do próprio ambiente de trabalho por meio da adoção de metodologias reflexivas e problematizadoras que proporcionem real transformação da prática ${ }^{6}$.

As ferramentas de comunicação como o Telessaúde, a Comunidade de Práticas e as propostas de $\mathrm{EaD}$ são recursos virtuais de aprendizagem estimulados no escopo da avaliação do PMAQ-AB que devem integrar iniciativas institucionais a fim de oportunizar qualificação e sistematizar tecnologias da gestão e do cuidado ${ }^{4,25}$.

O potencial dialógico desses dispositivos é reconhecido, mas iniciativas de ordem estrutural devem ser consideradas para que contemplem a existência de espaços que estejam de acordo com a realidade e as necessidades das equipes, haja vista o desenvolvimento da EPS e a qualidade do SUS ${ }^{25}$.

Considerado uma inovação na prática de gestão e associado à EPS, o apoio institucional constitui-se numa estratégia que aposta no trabalho coletivo, operando como disparador e facilitador de processos de trabalho a partir do estímulo ao exercício de autoanálise das equipes, fortalecendo os movimentos de mudança ${ }^{4}$.

A EP está pautada em diferentes perspectivas metodológicas, de modo a incorporar o aprender e o ensinar ao cotidiano do trabalho. Como os problemas de saúde precisam ser analisados e contextualizados mediante a prática social, os processos de EPS precisam ser incentivados pelas esferas estaduais e municipais ${ }^{4,6,25}$.

O PMAQ tem como pressuposto incentivar os profissionais e gestores da ESF a melhorar a qualidade dos serviços de saúde a partir da organização do trabalho e da reflexão crítica das práticas cotidianas. Nesse sentido, o programa não trata a EPS apenas como participação em cursos, mas sim como uma estratégia de gestão com potencial transformador das ações dos sujeitos e de suas práticas de saúde.

\section{CONCLUSÃO}

Os estudos em síntese apresentados nesta revisão destacam as experiências em relação à avaliação do PMAQ, as ações referentes à EPS inseridas no processo de trabalho das EqSF e o grau de incorporação das TIC na AB. Salienta-se a importância do PMAQ para o aperfeiçoamento das ações desenvolvidas pelos serviços, sendo a avaliação externa capaz

7 REVISTA BRASILEIRA DE EDUCAÇÃo MÉDICA

7 44 (1) : e010; 2020 
de oportunizar momentos de reflexão crítica sobre os processos de trabalho por parte dos profissionais da $\mathrm{AB}$, contudo são imprescindíveis o envolvimento e a mobilização de toda equipe de saúde e da gestão a fim de romper com o estigma negativo associado à cultura avaliativa.

Ainda há evidências do uso de práticas educativas formativas e verticalizadas que não levam em consideração as necessidades do território nem as demandas dos profissionais no cotidiano do trabalho em saúde, e percebe-se a fragilidade da inserção das TIC, principalmente, em territórios mais distantes dos centros urbanos, sendo estas consideradas estratégias potentes para o planejamento das ações e conseguinte melhoria na qualidade da atenção à saúde dos usuários.

A presente revisão aponta que há escassez de produções científicas referentes ao tema no Brasil. Sendo assim, permanece evidenciada a lacuna do conhecimento, pois parece não existir, de acordo com a busca realizada até o presente momento, uma pesquisa que retrate estratégias de EP no que se refere às dinâmicas inseridas nas práticas cotidianas das equipes para efetivação do processo avaliativo fomentado pelo PMAQ. Conclui-se que há muito ainda a se pesquisar na área.

\section{REFERÊNCIAS}

1. Brasil. Ministério da Saúde. Programa Nacional de Melhoria do Acesso e da Qualidade da Atenção Básica (PMAQ). Secretária de Atenção Básica à Saúde. Departamento de Atenção Básica. Brasília: Ministério da Saúde; 2011.

2. Cavalcanti PCS, Oliveira Neto AV, Sousa MFS. Uma narrativa sobre o Programa Nacional de Melhoria do Acesso e da Qualidade na Atenção Básica. In: Gomes LB, Barbosa MG, Ferla AA, organizadores. Atenção básica: olhares a partir do Programa Nacional de Melhoria do Acesso e da Qualidade (PMAQ-AB). Porto Alegre: Rede Unida; 2016 [acesso em 4 abr 2018]. Disponível em: http://historico.redeunida.org.br/ editora/biblioteca-digital/serie-atencao-basica-e-educacao-na-saude/ atencao-basica-olhares-a-partir-do-programa-nacional-de-melhoriado-acesso-e-da-qualidade-2013-pmaq-ab-epub.

3. Brasil. Ministério da Saúde. Portaria no 1.654, de 19 de julho de 2011. Brasília: Ministério da Saúde; 2011.

4. Brasil. Ministério da Saúde. Secretaria de Atenção à Saúde. Departamento de Atenção Básica (DAB). Programa Nacional de Melhoria do Acesso e da Qualidade da Atenção Básica (PMAQ): Manual instrutivo para as equipes de atenção básica e NASF. Terceiro ciclo (2015-2017). Brasília: Ministério da Saúde; 2017.

5. Brasil. Ministério da Saúde. Portaria $\mathrm{n}^{\circ} 1.645$, de 2 de outubro de 2015. Brasília: Ministério da Saúde; 2015.

6. Ribeiro DT, Nascimento DT do, Cunha FM da, Ozorio JC, Ferreira AV, Santos TC dos et al. O PMAQ-AB como umas das estratégias de estímulo à prática da educação permanente em saúde. In: Gomes LB, Barbosa MG, Ferla AA. A educação permanente em saúde e as redes colaborativas: conexões para a produção de saberes e práticas. Porto Alegre: Rede Unida; 2016 [acesso em 18 abr 2018]. Disponível em: https://www.lume.ufrgs.br/bitstream/ handle/10183/134335/000988700.pdf?sequence $=1$.

7. Moreira KS, Lima CA, Vieira MA, Costa, SM. Educação permanente e qualificação profissional para a atenção básica. Saúde e Pesquisa 2017 jan./ abr:10(1):101-9 [acesso em 4 abr 2018]. Disponível em: http://periodicos. unicesumar.edu.br/index.php/saudpesq/article/view/5682/3010.
8. Lopes EAA, Scherer MDA, Costa AM. O Programa Nacional de Melhoria do Acesso e da Qualidade da Atenção Básica e a organização dos processos de trabalho. Tempus (Brasília). 2015 jun;9(2):237-50 [acesso em 4 abr 2018]. Disponível em: http://www.tempusactas.unb. br/index.php/tempus/article/download/1757/1441

9. Jales ED, Jales RD, Gomes JGN. O PMAQ-AB enquanto ferramenta de gestão do trabalho em saúde a partir das percepções dos gestores e profissionais de saúde. Rev Saúde Ciênc 2017;6(3): 77-97 [acesso em 7 maio 2018]. Disponível em: http://www.ufcg.edu.br/ revistasaudeeciencia/index.php/RSC-UFCG/article/view/486/309

10. Jesus AS, Cardoso TSG, Vilela ABA, Nery AA. O enfermeiro no contexto do Programa Nacional de Melhoria do Acesso e da Qualidade da Atenção Básica (PMAQ): relato de experiência. Rev Saúde Com 2015;11(2):193-200 [acesso em 18 abr 2018]. Disponível em: http:// periodicos2.uesb.br/index.php/rsc/article/view/362/292

11. Campos FCC, Faria HP, Santos MA. Planejamento e avaliação das ações em saúde. Belo Horizonte: Nescon, UFMG; 2010.

12. Galvão TF, Pereira MG. Revisões sistemáticas da literatura: passos para sua elaboração. Epidemiol Serv Saúde 2014;23(1):183-4 [acesso em 12 ago 2019]. Disponível: http://www.scielo.br/scielo. php?script=sci_arttext\&pid=S2237-96222014000100183.

13. Galvão TF, Pansani TSAD, Harrad D. Principais itens para relatar revisões sistemáticas e meta-análises: a recomendação PRISMA. Epidemiol Serv Saúde 2015;24(2) [acesso em: 16 ago 2018]. Disponível em: http://www.scielo.br/scielo.php?script=sci_ arttext\&pid=S2237-96222015000200335

14. Silva LM, Ferreira LR, Rosa AS, Neves VR. Implementação do Programa de Melhoria do Acesso e Qualidade segundo gestores da atenção básica de São Paulo. Acta Paul Enferm 2017;30(4):397-403 [acesso em 6 dez 2018]. Disponível em: http://www.scielo.br/pdf/ape/ v30n4/0103-2100-ape-30-04-0397.pdf

15. Santos AF, Fonseca Sobrinho D, Araujo LL, Procópio CSD, Lopes EASL, Lima AMLD de et al. Incorporação de tecnologias de informação e comunicação e qualidade na atenção básica em saúde no Brasil. Cad Saúde Pública 2017;33(5):e00172815 [acesso em 6 dez 2018]. Disponível em: http://www.scielo.br/scielo.php?script=sci_ arttext\&pid=S0102-11X2017000505003\&lng=pt\&tlng=pt

16. Bispo Júnior JP, Moreira DC. Educação permanente e apoio matricial: formação, vivências e práticas dos profissionais dos Núcleos de Apoio à Saúde da Família e das equipes apoiadas. Cad. Saúde Pública 2017;33(9):e00108116 [acesso em 6 dez 2018]. Disponível em: http:// www.scielo.br/scielo.php?script=sci_arttext\&pid=S0102-311X201700 0905010\&lng=pt\&tlng=pt

17. Almeida JRS, Bizerril DO, Saldanha KGH, Almeida MEL de. Educação permanente em saúde: uma estratégia para refletir sobre o processo de trabalho. Rev. ABENO. 2016;16(2):7-15 [acesso em 8 dez 2018]. Disponível em: https://revabeno.emnuvens.com.br/revabeno/ article/view/248/209

18. Sampaio J, Moraes MN de, Marcolino EC, Castro, ID de, Gomes, LB, Clementino FS et al. PMAQ-AB: a experiência local para a qualificação do Programa Nacional. Rev. Enferm UFPE 2016; 10(Supl. 5):4318-28 [acesso em 22 out 2018]. Disponível em: https://periodicos.ufpe.br/ revistas/revistaenfermagem/article/download/11179/12720.

19. Machado JFFP, De Carli AD, Kodjaoglanian VL, Santos MLM dos. 
Educação permanente no cotidiano da atenção básica no Mato Grosso do Sul. Saúde Debate 2015;39(104):102-13 [acesso em 22 out 2018]. Disponível em: http://www.scielo.br/scielo.php?pid=S0103$11042015000100102 \& s c r i p t=s c i \_a b s t r a c t \& t \operatorname{lng}=e s$

20. Pinto HA, Ferla AA, Ceccim RB, Florêncio AR, Matos IB, Barbosa MG et al. Atenção básica e educação permanente em saúde: cenário apontado pelo Programa Nacional de Melhoria do Acesso e da Qualidade da Atenção Básica (PMAQ-AB). Divulg Saúde Debate 2014;(51):145-60 [acesso em 8 dez 2018]. Disponível em: http://cebes. org.br/site/wp-content/uploads/2014/12/Divulgacao-51.pdf

21. Brasil. Ministério da Saúde. Secretaria de Atenção à Saúde. Departamento de Atenção Básica (DAB). Programa Nacional de Melhoria do Acesso e da Qualidade da Atenção Básica (PMAQ): Autoavaliação para melhoria do acesso e da qualidade da atenção básica: AMAQ. Brasília: Ministério da Saúde; 2017.

22. Andrade RTS de, Santos AM dos, Oliveira MC. Programa de Melhoria de Acesso e Qualidade da Atenção no Município de Amargosa, Bahia. Rev Baiana Saúde Pública 2016;40(1):123-46 [acesso em 10 jun 2018]. Disponível em: http://pesquisa.bvsalud.org/portal/resource/pt/ biblio-859616.

23. Moreira, KS, Vieira, MA, Costa, SM. Qualidade da atenção básica: avaliação das equipes de saúde da família. Saúde Debate 2016;40(111):117-27 [acesso em26 set 2018]. Disponívelem:http://www. scielo.br/scielo.php?pid=S0103-11042016000400117\&script $=$ sci abstract\&tlng=pt.

24. Brasil. Ministério da Saúde. Secretaria de Atenção à Saúde. Departamento de Atenção Básica (DAB). Autoavaliação para Melhoria do Acesso e da Qualidade da Atenção Básica - Núcleos de Apoio à Saúde da Família AMAQ - NASF. Brasília: Ministério da Saúde; 2013.
25. Bezerra, MM, Medeiros KR. Limites do Programa de Melhoria do Acesso e da Qualidade da Atenção Básica (PMAQ-AB): em foco, a gestão do trabalho e a educação na saúde. Saúde Debate 2018;42(núm. esp. 2):188-202 [acesso em 7 jan 2019]. Disponível em: http://www.scielo.br/scielo.php?script=sci_abstract\&pid=S0103$11042018000600188 \& \operatorname{lng}=\mathrm{en} \& \mathrm{nrm}=\mathrm{iso} \& \operatorname{lng}=\mathrm{pt}$.

\section{CONTRIBUICÃO DOS AUTORES}

Livia Mendes Mesquita: mestranda do Mestrado Profissional em Ensino na Saúde, responsável pelo projeto, participou de todo planejamento, da construção científica, execução e confecção do manuscrito.

Prof ${ }^{\mathrm{a}}$. Dr ${ }^{\mathrm{a}}$ Geilsa Soraia Cavalcanti Valente: orientadora e supervisora do projeto, participou da construção científica do manuscrito, da redação e das etapas de leitura e revisão.

Prof ${ }^{a}$. Dr ${ }^{a}$ Elaine Antunes Cortez: participou das etapas de leitura e revisão.

Raquel Lima Soeiro, Simone Costa da Matta Xavier e Bianca Maria Innocencio da Silveira Lobo: mestrandas do Mestrado Profissional em Ensino na Saúde, participaram das etapas de leitura e revisão.

\section{CONFLITO DE INTERESSES}

As autoras declaram não haver conflitos de interesse neste estudo.

\section{ENDEREÇO PARA CORRESPONDÊNCIA}

Rua Santa Rosa, nº 146 / apto 1201 - Icaraí - Niterói, Rio de Janeiro-

RJ - CEP: 24220-420.

mmesquita.livia@gmail.com 